\title{
Factors of radial bone strength after marginal defect formation
}

\author{
N.M. Aleksandrov', V.D. Veshutkin ${ }^{2}$, A.E. Zhukov², I.D. Veshaev ${ }^{1}$, D.A. Kuptsov' ${ }^{1}$, O.I. Uglev ${ }^{1}$ \\ ${ }^{1}$ Privolzhsky Research Medical University, Nizhny Novgorod, Russian Federation \\ ${ }^{2}$ Nizhny Novgorod State Technical University n.a. R.E. Alekseev, Nizhny Novgorod, Russian Federation
}

\begin{abstract}
Purpose To determine the effect of biometrical parameters of the radial bone and due to edge defect formed on the radius strength properties using calculation methods. Materials and methods The study of bone strength affecting factors was conducted with the aid of experimental and calculation methods. Biometrical parameters were studied in 10 pairs of the human cadaveric radius as an intact bone initially and after the formation of rectangular or triangle-shaped edge cuts. To determine the stress-strain behaviour, mathematical calculations were performed based on the beam flexural theory for isotropic materials. Computation study were conducted using the finite element method with the NX Siemens software package. Based on assumed mathematical models, the actual areas of safe loads in the presence of cuts and values of destructive loads depending on the depth and shape of a cut taking into account the initial curvature of the bone as well as the criteria of a required residual strength in variation of influencing parameters were identified by means of calculations. Results It was established that an increase in bone curvature results in the reduction of longitudinal destructive loads and in increasing values of the normal strength. The 0.05 bone curvature combined with the 0.5 cut causes a decrease in the ultimate load by 20 times (up to $4.8 \%$ for a rectangular cut and to $5.4 \%$ for a triangular cut). A 0.5 -deep cut in the bone which curvature is 0.05 enhances the normal stress by 6.9 times for a triangular cut and by 7.8 times for a rectangular one as compared to a bone without curvature. The critical values for the curvature and depth of the cut were established which permit to avoid additional bone reinforcement. Conclusion The strength of the radius with a maginal defect depends not only on the depth of a cut but on its location, shape and on the radius curvature.
\end{abstract}

Keywords: osteocutaneous radial flap, radial bone defect, radial bone strength, experiment, calculation, computer simulation

\section{INTRODUCTION}

Animportantachievement of currentreconstructive and restorative surgery is the development of precision technologies for the use of free grafts and non-free vascularized osteocutaneous grafts $[1,2]$. The use of such tissue complexes enables to achieve qualitatively new treatment results in many areas of reconstructive surgery, since adequate blood supply, primary union of bone fragments, their resistance to resorption as well as soft tissues recovery are thus provided, what is especially important in the elimination of tissue defects in various anatomical areas [1-7]. However, such interventions inevitably lead to the formation of a marginal bone defect and are associated with donorsite morbidity, the management of which is a difficult problem and often worsens treatment results $[8,9]$.

Donor-site defects are especially large if the interventions are in the maxillofacial area as it is associated with the need to collect a significant volume of tissues [10]. Bone defects in the bearing donor tubular bone are most serious in terms of functional or cosmetic deficiency [11]. The radius is often used as a donor bone for surgical reconstruction in various parts of the musculoskeletal system [7]. In maxillofacial surgery, plasy with a radial osteocutaneous graft has been currently recognized as the "gold standard" of treatment $[12,13]$. The reasons are the possibility of creating a long vascular pedicle and a sufficient diameter of its artery and veins for reliable anastomoses for good blood supply to the bone graft and the soft tissues surrounding it. The graft is harvested together with soft tissues without disrupting the continuity of the bone. However, the collection of such a graft is naturally associated with a marginal bone defect that might cause a pathological fracture $[14,15]$. Its management is an extremely difficult problem, often comparable to that of the actual reconstructive intervention in the recipient area due to disorders in blood circulation, disintegrity of the periosteum and bone deficiency. The incidence of pathological fractures has been currently not decreasing and reaches $18 \%$. Thus, it may cause significant cosmetic and functional disorders of the limb and prolonged treatment period [16-21].

The strength properties of the radius due to a marginal defect have been insufficiently studied. Thus, only external destructive loads have been assessed and the only biometric indicator is the permissible depth of bone cut obtained in the experiment without taking into account physiological loads and the use of precise calculation methods $[22,23]$. The evidence base for these studies is not always sufficient [24]. Moreover, the bone has been studied mainly for bending and rotational loads [25], assuming that a bone fracture occurs only under these types of stress. This is due to the fact that experimental approaches do not provide investigation of all strength factors due to limited possibilities of sampling the material under study and its extreme variability. It is difficult to form comparison groups that are homogeneous in biometric parameters. It is unrealistic to select the bones with the necessary 
and identical biometric parameters for experimental studies in order to assess the influence of various factors and, especially, their combinations on bone strength. Due to individual differences in bone parameters, a large amount of cadaveric material is required to form homogeneous comparison groups, what is practically impossible. Moreover, the elasticity modulus of bones change significantly depending on gender, age, nature of the profession, concomitant pathology, etc. Due to these circumstances, the importance of mathematical modeling grows with the involvement of the technical beam flexural theory and other calculation methods in the study of material strength, structural mechanics, as well as modern numerical methods (finite element method) for evaluating the strength (bearing) properties of the donor radius [26].

However, only a few works describe the use of accurate mathematical methods to assess the internal elastic forces arising in the distal part of the radius under loading. Thereby, good agreement was noted between the calculated and experimental data [27, 28]. Internal force factors (elastic forces) that arise during various types of loading of the intact radius and the one with a marginal defect remain unexplored. Only the depth of the cut for graft harvesting was taken into account to assess the residual strength of the radius $[21,23]$. Other biometric parameters (the shape of the cut, its location, the initial curvature of the bone) practically remain unconsidered. Some works were devoted to the influence of the radius and ulna curvature on their strength properties, but they studied intact bones. Currently, there are no works on the study of the dependence of the strength of the radius with a marginal defect on its bending stresses arising under the action of a longitudinal tension-compression load in the presence of the initial curvature of the bone. Measures for prevention of pathological fractures include only recommendations not to exceed the depth of the cut to more than $1 / 3$ of the bone diameter. At a greater depth of the cut, it is recommended to perform bone reinforcement interventions such as bone grafting and osteosynthesis with a plate on screws $[9,21,29]$. The indications for osteosynthesis in fractures of the forearm bones and the choice of options for their fixation consider only the nature of the fracture and the state of the bone fragments. Other biometric parameters are not taken into account. Therefore, we conducted the numerical studies on the influence of various biometric factors and options for enhancing the strength of the bone with its marginal defect and determined their critical values using the technical flexural beam theory and the method of computer modeling.

Purpose To determine the effect of biometrical parameters of the radius and its marginal defect on the strength properties of this bone using calculation methods.

\section{MATERIAL AND METHODS}

The factors affecting bone strength were studied with experimental and calculation methods. The biometric parameters of the intact radius and the radius after marginal cuts on the lateral surface of its distal half were studied in an experiment on 10 pairs of human cadaveric radial bones. The size of the cut on the bone corresponded to the average size of defects formed after graft collection for reconstructive operations on the hand (osteocutaneous flaps for reconstruction of fingers management of bone defects) and other anatomical areas. The bone was sawn in the transverse direction in the middle of the defect. In intact bones, the area at a similar level where the graft should be taken (in the projection of the imaginary defect) was sawn to determine the cross-sectional shape.

In addition, the bones were cut through in the middle of the proximal part. The dimensions of the cross-section of the bone were measured: width, height, layer thickness. The radius of the bone curvature was assumed to be equal to the half of the section width. For the section with the defect notch, the residual width, height and thickness of the bone section were measured. For determining the cross-sectional area of the bone with a defect cut notch, the width and height of the cross-section were measured, and the radius of the curvature was measured when an intact bone (without a cut notch) was transected. For further calculations, the mean values of the cross-sectional dimensions were taken, obtained from measurements of fourteen natural radial bones. The obtained measurement data were used to calculate the geometric characteristics of the cross-section (cross-sectional area, axial moments of inertia of the area, coordinates of the center of gravity of the cross-section), which were necessary for further calculations of the strength. The initial curvature of the radius was measured in two planes on 10 pairs of human cadaveric radial bones. To do this, the radius was applied to the horizontal plane, and the distance between the horizontal plane and the upper or lower edge of the bone was measured using a millimeter ruler (or caliper). Such measurements were performed on both sides of the middle of the bone at a certain distance to study the distribution of the curvature along the length. Based on the analysis of the measurement results, the distribution function of dimension-free curvature along the length shaped as a sinusoid was adopted.

As a result of the analysis of experimental measurements, the shape of the bone cross-section was conventionally taken as the shape shown in Figure 1 for a cross-section without a cut notch. Figure 2 shows the resulting sectional shape in the cut notch zone, when its depth is more than the bone radius, that is, $h_{0} \geq R=B / 2$. 


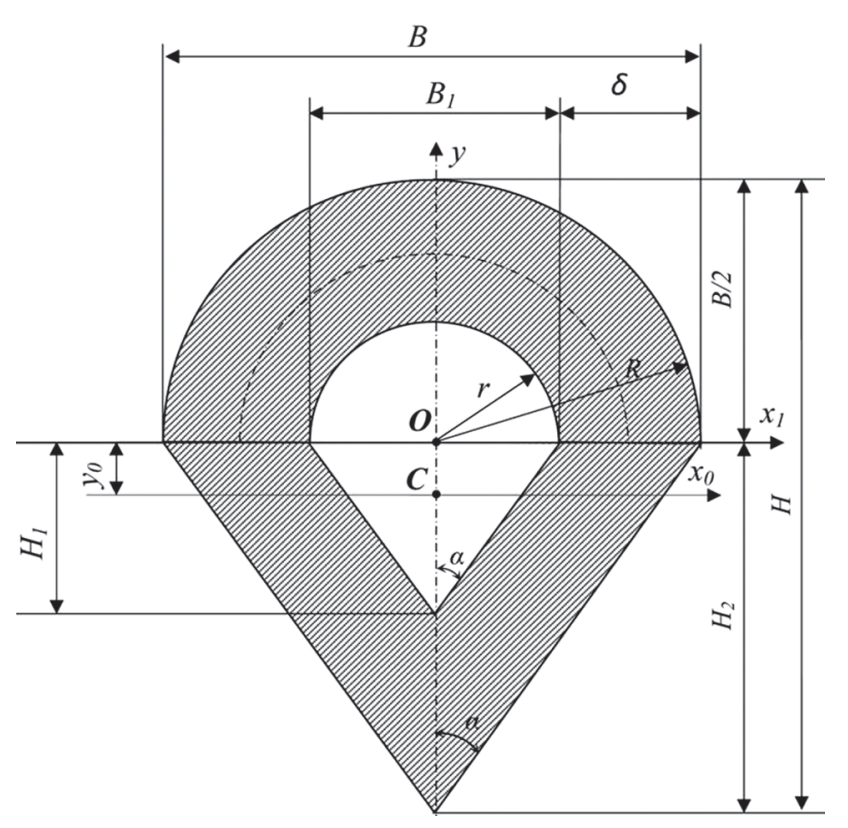

Fig. 1 The shape and designations of the dimensions of the intact section of the bone: $\mathrm{x} 1$ axis passing along the line of conjugation of the triangular and radial elements; $y, x$ are the central axes of the cross section (y-axis is the axis of symmetry of the section); $H$ is the height of the crosssection of the intact bone; $B$ is the width of the cross-section of the intact bone; $B_{l}$ is the width of the internal cavity of the bone; point $C$ is the center of gravity of the cross section

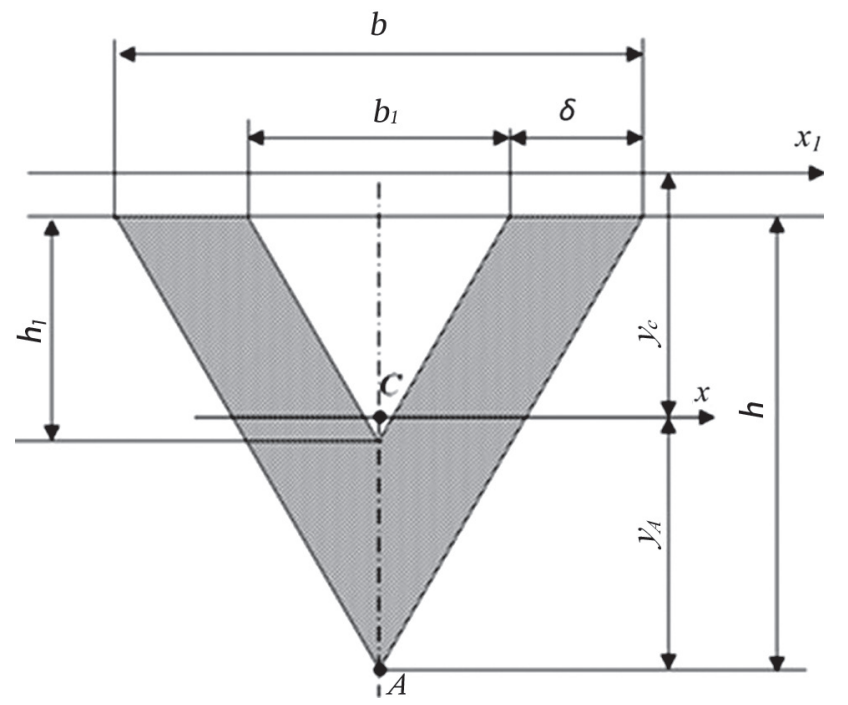

Fig. 2 The shape of the section in the notch zone (section D) and the designation of the dimensions: $\mathrm{x} 1$ is the axis passing along the line of conjugation of the triangular and radial elements; $x, y$ are central axes of the cross-section ( $y$-axis is axis of symmetry of the section); $h$ is the height of the cross-section of the intact bone; $b$ is the width of the cross-section of the intact bone; $b$ is the width of the inner cavity of the bone; $\delta$ is the thickness of the force layer; point $C$ is the center of gravity of the cross-section

Thus, the elements that describe the section shape are the width, height and thickness of the profile. This simplified shape makes it much easier to calculate the geometric characteristics of the cross-sectional area of the profile (area, axial moments of inertia, moments of resistance and the position of the center of gravity of the section).

It is obvious that it is almost impossible to conduct in-situ experiments in clinical settings. Therefore, the use of analytical methods to determine the internal force factors (elastic forces) acting on the bone is quite justified. Mathematical calculations to determine the stress-strain state were carried out on the basis of the technical theory of bending moment. Due to the fact that the marginal defect is located in the area of the diaphysis, where the orientation of osteons is longitudinal, the anisotropy of the bone may be neglected and the bending theory can be applied. Considering the fact that the marginal defect of the human radius definitely cannot be not filled in the course of life, the factor of bone regeneration may also be neglected. The study of the influence of depth, shape of the defect cut and initial bending of the radius on its strength properties (normal stresses, ultimate breaking loads) in tension, torsion and bending was conducted. The residual bone strength for various depths of defects under experimental distraction (compression), bending physiological loads and torsion was assessed.

The experimental studies [30] revealed the effect of bone curvature on its deformation and internal force factors (longitudinal bending moments, normal stresses) under compressive and tensile loads, which served as the basis for the application of formulas for determining normal stresses in a section under eccentric tension-compression.

The position of the neutral line $(N L)$ and the point of intersection are shown in Figure 3.

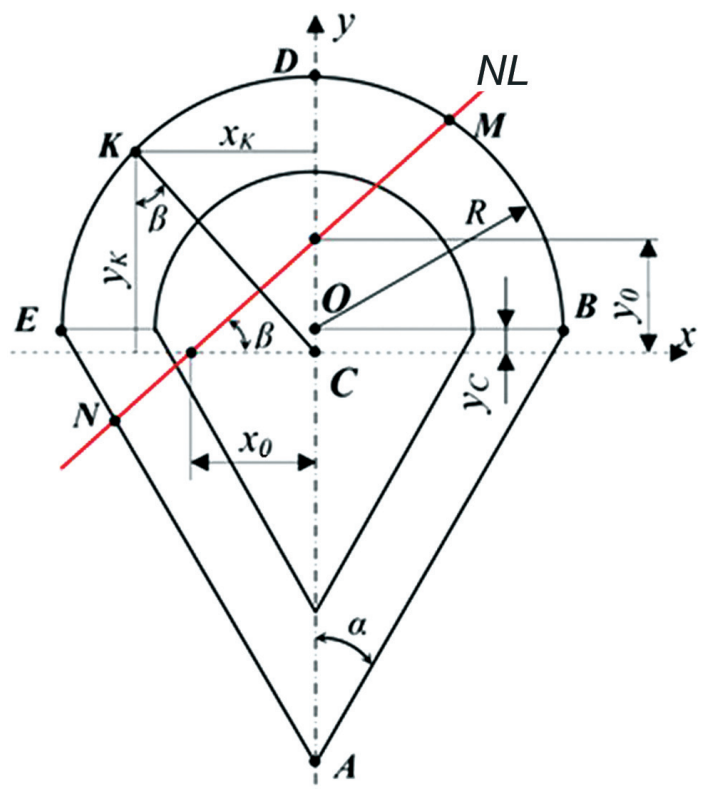

Fig. 3 Position of the neutral line (NL) and dangerous points of the bone section without cut notch under tension

The part of the section located below the $N L$ is in the tension zone, and the part of the section above the $N L$ is in the compression zone. The most loaded point is point $A$ in the tension zone, and point $K$ in the compression zone, as the most distant ones from the $N L$ (Fig. 3).

Normal stress $\sigma$ arises in a deformed body under the influence of various factors, is a measure of internal forces of separation or compression per unit of area and directed perpendicular to the section of the element. 
Under tension, the total normal stresses were determined by the following formula (1) for any point of the cross-section:

$$
\sigma=\frac{N}{A}-\frac{M_{x}}{I_{x}} \cdot y_{i}+\frac{M_{y}}{I_{y}} \cdot x_{i},(1)
$$

where $N$ is the longitudinal force in the section; $A$ is crosssectional area; $y_{i}$ is the coordinate of the section point, where the mechanical stress is determined relative to the neutral axis $x, M_{x}$ is the bending moment relative to the axis $x ; I_{x}$ is the moment of inertia of the cross-sectional area relative the central axis $x ; x_{i}$ is the coordinate of the section point, where the mechanical stress is determined, relative to the neutral $y$-axis, $M_{y}$ is the bending moment relative to the $y$-axis; $I_{y}$ is the moment of inertia of the cross-sectional area relative to the central axis $y$.

On the basis of the formula (1), formulas were derived for determining the normal stresses $\sigma_{z}$ and external breaking loads $P_{1}$ under eccentric tension (compression) taking into account the existing cut of the bone and its curvature in two planes [30].

Previous experimental studies confirmed the results obtained with the formulas developed. It indicates the adequacy of the mathematical model [30]. Using the obtained dependencies, a series of calculations was performed to assess the influence of the initial bone curvature, shape and depth of the cut on bone strength characteristics.

The computational method allows one to study the effect of the initial bone curvature and depth of the cut notch at various combinations of these parameters under the conditions of its constant characteristics (breaking stress, modulus of longitudinal elasticity) on the stress-strain state (SSS) of the bone. In the computational models, bones of different cut shapes and sizes, initial curvatures were given the same characteristics of the bone material for the possibility of studying the effect of each parameter: breaking stress in bending and tension $\sigma_{w}=120 \mathrm{MPa}$, Young's modulus (longitudinal elasticity) $E=2 \times 10^{4} \mathrm{MPa}$. To obtain reliable results, the studied bone models differed only in the parameter studied, while other characteristics of the bones were the same. Numerical data were obtained with a longitudinal force $N=15 \mathrm{~kg} \approx 147 \mathrm{H}$, what corresponds to the average weight lifted by a person. These parameters coincide with the loads known in the literature and applied in the experiment [31].

Transverse bending moments arising under physiological loads on the forearm were calculated on the basis of literature data [32-34] to determine the effect of the shape and location of the bone cut on its strength properties. Taking these data into account, diagrams of the distribution of transverse bending moments under physiological and experimental loads were constructed for rectangular and triangular cutouts [30]. After plotting the diagrams of bending moments, dangerous sections of the bone were determined.

The numerical method $[35,36]$ enables to verify the proposed analytical calculation method, to obtain the superficial and internal distribution of normal stresses and deformations, and also to evaluate the stability of the bone with the determination of critical loads in compression for various types of stability failure [37].

The finite element method (FEM), that recently has become widely used, was applied to calculate the strength of the bone $[14,37]$. The essence of the method is to construct a geometric model with its subsequent breakdown into geometrically simple bodies, or elements, for each of which an equilibrium equation can be written, and all equilibrium equations are solved simultaneously.

To determine the stresses and displacements, calculations were performed using the FE (finite element) method [36] in the NX Siemens software system.

The NX brings together key features to quickly, efficiently, and flexibly use numerical simulation tools for strength calculations and interdisciplinary physical analysis. Simulation and further calculations were also done with the NX Siemens software.

The geometric model is a cylindrical structure with a cross-section composed of a semi-circle and two lateral trapezoids, symmetrical relative to the common axis. The following geometrical models were developed for the study: a model with a straight axis; a model with curvature in two planes; a model with rectangular and triangular cutouts with a straight axis; a model with curvature in two planes with rectangular and triangular cutsouts with a depth of 0.16 and 0.33 .

To study the stress-strain state of the radius in its preventive fixation and dependence on the shape of the artificial cut, the thickness and shape of the metal plates, the depth of screw immersion into the bone, bone assemblies were simulated from a model with curvature in two planes and rectangular and triangular cutouts with a depth of $h_{\delta} / H=0.33$, one- or two-millimeter thick plates of various shapes and widths, with screws immersed to 4 $\mathrm{mm}, 13 \mathrm{~mm}$ and $18 \mathrm{~mm}$. We also analyzed the behavior of a bone model with a compensating plate under a compressive load to determine the types of instability. Loading of the computational model was produced by pressure at a free end along the cross-sectional surface, as well as at the node of fixing the faces with moment. The applied tensile load is $\mathrm{P}=981 \mathrm{~N}$, and the applied single moment is $\mathrm{M}=100 \mathrm{Nm}$. Upon completing tensile and torsion calculations with the NX Siemens software, the stress and displacement fields of the test samples were obtained.

\section{RESULTS}

Strength properties of the bone in transverse bending Analysis of the bending moment epure diagrams showed that the dangerous section of the triangular cutout is located at a distance $z / l_{0}=0.342$ 
(from the distal end of the radius), the bending moment in which is equal to $M_{l}=0.43 \mathrm{Nm}$; the dangerous section of the rectangular cutout is located at the distance $z / l_{0}=0.51$, the bending moment in which is equal to $M_{2}=2.25 \mathrm{Nm}$. The dangerous section of the triangular cutout is in the zone of small moments under the action of physiological loads in contrast to the dangerous section of the rectangular cutout which is in the zone of medium loads. The bending moment in the dangerous section of a rectangular cut is 5.2 times greater than the bending moment in the dangerous section of a triangular cut (normal stresses also differ by 5.2 times). Therefore, by optimizing the location of the dangerous section in transverse bending, the acting stresses may be reduced by up to 5 times. Studies have shown that a triangular cut is more favorable in terms of the perception of bending load. In a transverse physiological bending, the relative permissible depth of a triangular cut should not exceed 0.49 , and of a rectangular cut should not exceed 0.22.

Strength properties of the bone under axial loads Criteria for the permissible residual strength due to the action of longitudinal forces were also obtained; graphs were proposed for determining the permissible depth of triangular and rectangular cuts according to the measured parameters of the curvature of the bone and its cross-section.

The performed calculations of the residual strength of the donor radius with the determination of external and internal force factors acting on it enabled to obtain the following data. The relative breaking load, as an external force factor, was studied using the derived formulas [30] to determine the bearing capacity of the bone, taking into account its initial curvature, as well as the effect of the depth and shape of the cut notch on the strength of the bone. Based on the calculated data, the dependence of the breaking load on the initial curvature of the bone by its stretching in different depths and shapes of the cut was revealed. The influence of the initial curvature at a constant relative depth of the defect on the relative breaking load in tension was studied by the calculation method (Fig. 4 a).

The relative initial curvature was determined by the ratio $f_{0} l_{0}$, where $f_{0}=f_{y}$ is the value of the arrow of the initial curvature only in the $Y O Z$ plane, and $l_{0}$ is the calculated length of the bone. To simplify the analysis, the influence of the bone curvature in one direction $Y O Z$ was investigated at the average dimensions of the bone cross-section. The presence of the initial curvature $f_{x} / l_{0}$ in another plane $(X O Z)$ of the bone leads to a change in the stress state only at the points of the cross-section where the stresses are not maximum, provided that the initial curvature in the $X O Z$ plane does not exceed the curvature in the $Y O Z$ plane. The maximum normal stresses are achieved at point $A$, lying on the axis of symmetry (Fig. 3), therefore, the presence of initial curvature in the plane $(X O Z)$ does not lead to a change in the breaking loads and tension at this dangerous point. If $f_{x} / l_{0}$ is more than $f_{y} / l_{0}$, the stresses increase due to the move of the calculated point into the compressed zone (point $\mathrm{K}$ in Fig. 3). The analysis of geometric parameters performed on 10 paired bones showed that the condition $f_{x} / l_{0} \leq f_{y} / l_{0}$ is satisfied, and therefore, in the framework of this model, it may be ignored in most cases. Each curve on the graph (Fig. 4 a) characterizes a bone with a constant depth and shape of the cutout.

The graphs are given for triangular and rectangular cutouts at average cross-sectional dimensions. The relative breaking load $\bar{P}_{1}$ was determined by the ratio $P_{1} / P_{0}$, where $P_{0}$ is the breaking load of the bone without defect and without curvature, and $P_{1}$ is the breaking load of the bone with a given shape and depth of the defect and with variable curvature changing from zero to 0.05 . As follows from the graphs, the relative breaking stress decreases rapidly as the curvature increases. When the curvature changes in the range $f_{f} l_{0}=0 \ldots 0.015$, the stress drop is more significant, compared to the range $f_{d} l_{0}=0.015 \ldots 0.05$, where the stress drop is less significant. Therefore, even a small increase in the initial curvature leads to a significant reduction in breaking loads. Since the calculated point (dangerous) for the usual relations of the defect depth is, as a rule, on the axis of symmetry, then there is no influence of the relative initial curvature for this point in the XOZ plane.

The graphs presented allow us to determine the normal stresses in regard to curvature of the bone in both directions under the condition $\mathrm{f} x / 10<f_{y} / l_{0}$.

It was revealed that the maximum normal stresses depend linearly on the value of the initial curvature at a constant cut depth (Fig. 4 c). According to the graphs (Fig. $4 \mathrm{~b}$ and c), it is quite simple to determine the permissible depth of the cut for the given values of the initial curvature and the given level of permissible stresses. For intermediate values of the initial curvature, linear interpolation between the values taken from the graph is acceptable. The horizontal line corresponding to the level of permissible stresses will intersect the graph line with the given curvature. The intersection point will define the permissible depth of the triangle or rectangular cut.

For the variant without curvature and without cut notch (straight bone with constant cross-section), normal stresses are equal to $\sigma_{z}=1.47 \mathrm{MPa}$, are uniformly distributed over the section and correspond to simple tension (compression) without bone bending (Fig. 4, c). Normal stresses with a relative cut depth $h_{0} / H=0.5$ without curvature are equal to $\sigma_{z}=6.33 \mathrm{MPa}$. Thus, the stresses increased by 4.3 times. For a bone without a cut notch but with a curvature $f_{v} l_{0}=0.05$, the normal stresses are $\sigma=8.67 \mathrm{MPa}$, what corresponds to their increase by 5.9 times. Hence, it can be concluded that the initial 
curvature of the bone has a more significant effect on the magnitude of the maximum normal stresses than the presence of a cut notch.
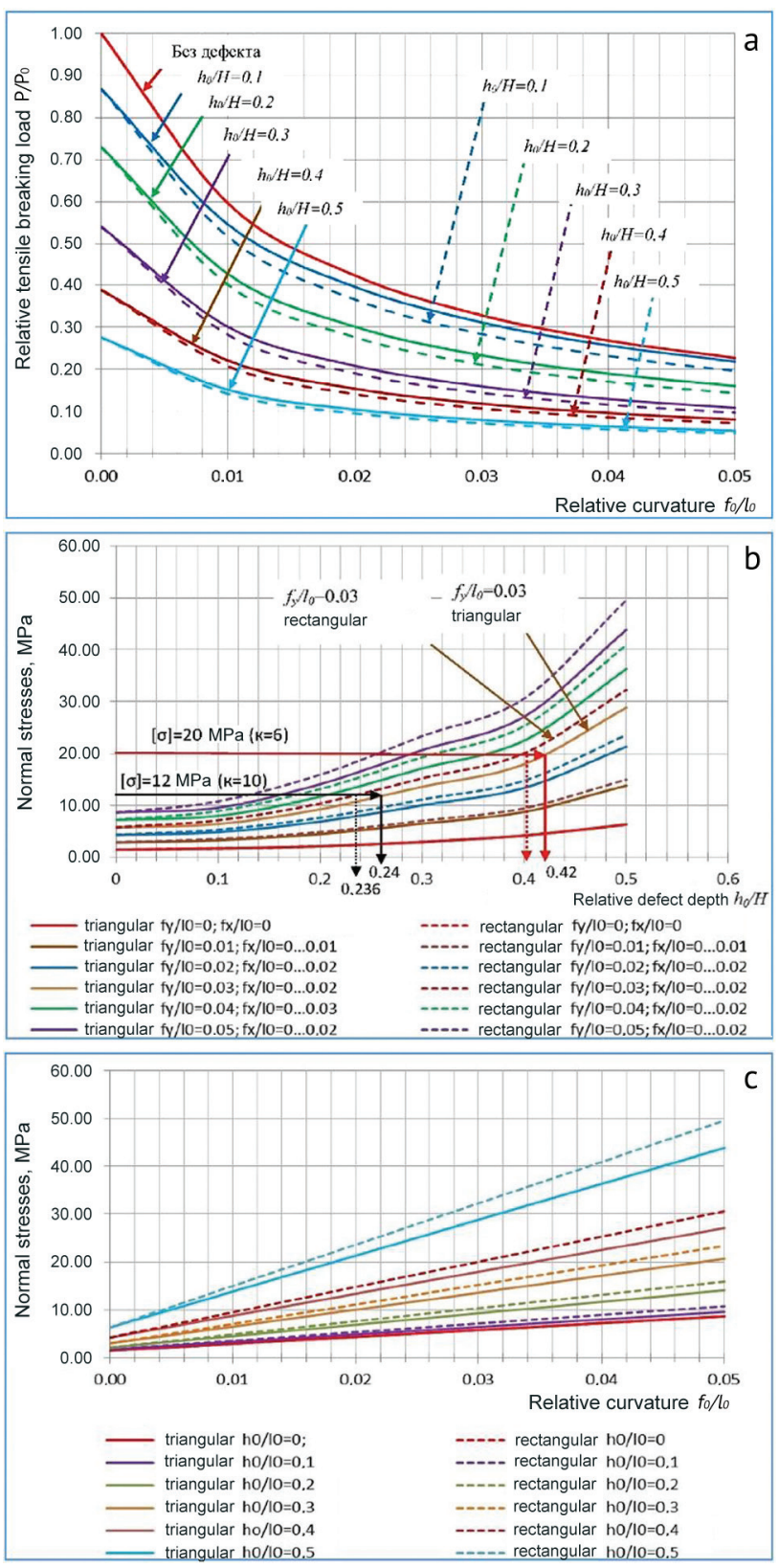

Fig. 4 Dependence: $\boldsymbol{a}$ the relative breaking load in stretching on the initial curvature of the bone in only one direction (in the $Y O Z$ plane) at a constant relative defect depth. Solid line - a triangular cut; dashed line - a rectangular cut; $\boldsymbol{b}$ normal tensile stresses in stretching on the depth of bone defect at constant relative curvature for different cut shapes; $\mathrm{c}$ normal stresses on the curvature in one direction at fixed values of the cut depth under tensile force of $15 \mathrm{kgf}$

Let us estimate the simultaneous effect of the curvature and cut notch on normal stresses. To do this, let us compare the normal stresses in the bone with a cut notch $h_{\delta} / H=0.5$ without curvature $\left(\sigma_{z}=6.33 \mathrm{MPa}\right)$ and with a curvature $f_{y} / l_{0}=0.05$. In this case, the normal stresses are $\sigma_{z}=43.82 \mathrm{MPa}$ for a triangular cutout and $\sigma_{z}=49.55 \mathrm{MPa}$ for a rectangular cutout. For a triangular cut, the normal stresses increased by 6.9 times, and for a rectangular cut by 7.8 times. Compared to a straight bone without a cut, the stresses increased by almost 30 times for a triangular cut notch and by 34 times for a rectangular one. From Figure $4 \mathrm{~b}$, it can be concluded that the normal stresses for each fixed curvature linearly depend on the relative depth of the cut. For example, as a result of bone measurements, the values $l_{0}=180 \mathrm{~mm}$, $f_{v}=5.4 \mathrm{~mm}$ were obtained. Then $f_{v} / l_{0}=0.03$, the permissible stresses are taken with a safety factor of $k=6$ and for the level of permissible stresses $[\sigma]=120 / 6=20 \mathrm{MPa} f_{y} / l_{0}=0.03$. The intersection points will correspond to the limit relative depth of the triangular cut $h_{\delta} / H=0.42$ and the limit relative depth of the rectangular cut $h_{\delta} / H=0.4$.

The resulting graphs allow one to determine the permissible loads at a relative cut depth of 0.3 recommended in the literature for various values of the radius curvature (Fig. 4 b).

With the depth of a rectangular defect of $h_{\delta} / H=0.3$ and slope of curvature in one direction $f_{0} l_{0}=0.05$, the normal stresses will be $23.0 \mathrm{MPa}$. If we assume normal stresses $[\sigma]=30 \mathrm{MPa}$, then the tensile or compressive load can be increased and allow $[P]=15 \times 30 / 23.0 \approx 19.6 \mathrm{~kg}(15 \mathrm{~kg}$ is the average load that a person can lift; $30 \mathrm{MPa}$ is permissible normal stress; 23.0 is a point on the curve at a depth of 0.3 for a curvature of 0.05). Under the conditions of similar curvature and depth of the triangular defect, the permissible load will be $22.5 \mathrm{~kg}$.

With the depth of a rectangular defect $h_{\delta} / H=0.3$ and curvature in one direction $\mathrm{f} 0 / 10=0.03$, the normal stresses will be 14.0 MPa. If we assume normal stresses $[\sigma]=30 \mathrm{MPa}$, then the tensile or compressive load can be increased and allow $[P]=15 \times 30 / 14.0 \approx 32.1 \mathrm{~kg}$. Given the same curvature and depth of the triangular defect, the permissible load will be $37.5 \mathrm{~kg}$.

Taking the breaking load for intact bone without curvature as $100 \%$, we can estimate the influence of the relative depth of the cut and the relative initial curvature on the value of the breaking load. If a cut is with a relative depth $h_{\delta} / H=0.5$ but without an initial curvature, the relative tensile load will decrease to $28 \%$. In a bone with a relative initial curvature $f_{y} l_{0}=0.05$ but without a cut notch, the breaking load drops to $23 \%$. In the presence of a cut $h_{0} / H=0.5$ and an initial curvature $f_{y} / l_{0}=0.05$, the relative breaking load will decrease for a triangular cuut to $5.4 \%$, and for a rectangular one to $4.8 \%$ (Fig. 4 a). Thus, a significant curvature of the bone in combination with a sufficiently deep cut leads to a 20 -fold decrease in the ultimate load.

It was revealed that the combination of the initial curvature in two directions does not lead to an increase in the stresses, as mentioned earlier, when the condition $f_{x} / l_{0}<f_{y} / l_{0}$ is satisfied, since the calculation point is located on the axis of symmetry. If the condition $f_{x} / l_{0}<f_{y} / l_{0}$ is not met, then the calculated point of the section is the point $\mathrm{K}$ (Fig. 3), and the 
curvature of the bone in the other direction increases the calculated stresses depending on the relative curvature in this direction.

Analysis of the data obtained shows that when the relative initial curvature $f_{x} / l_{0}$ is less than the relative curvature $f_{v} / l$, then it does not affect the maximum stresses. Analysis of all performed calculations also revealed that a triangular cut is more preferable from the point of view of residual bone strength, since the calculated section is located closer to the wrist joint, and the initial curvature has less influence on the magnitude of normal stresses compared to a rectangular cut.

Thus, both the depth and the curvature of the bone have impact on the strength of the radius with a marginal defect. The literature sources present only the permissible bone depth, equal to $1 / 3$ of the diameter, but it was calculated under experimental loads not regarding bone curvature. As our study has shown, both factors must considered by assessing bone strength. Due to a sharp increase in normal stresses depending on the cut depth and the curvature of the bone, it becomes necessary to determine the critical magnitudes of these parameters. The critical magnitude of the combination of curvature and cut depth is the smallest value of the combination of these parameters, at which a sharp rise in normal stresses is noticeable, and as a result, the probability of fracture increases when the bone is in tension.

The safety factor for various structures, according to the literature, is $2-5$. Taking into account the presence of a bone cut, a high stress concentration factor, which significantly reduces bone strength, and also taking into account the unforeseen loads in case of injuries, the safety factor " $k$ " is taken by us to be from 6 to 10 in tension and $k=4$ in bending (the condition of strength equality of the damaged and intact bone). A larger value of the coefficient should be taken based on the condition of the whole bone, as well as the patient. The calculation was performed for two values of the safety factor $(k=6$ and $k=10$ ), which is reflected in Figure 5 ( $\mathrm{a}$ and $\mathrm{b}$ ). The maximum allowable cut depths with known curvature values were studied for an allowable stress of $12(k=10)$ and $20(k=6) \mathrm{MPa}$. Using the given graphs (Fig. $5 \mathrm{a}, \mathrm{b}$ ), the permissible depth for triangular and rectangular cuts can be determined for any value of bone curvature in two planes.

Figure $5 \mathrm{a}$ shows graphs of the change in the curvature $f_{y} l_{0}$ in the range from 0 to 0.08 with a step of 0.005 depending on the relative depth of the cut in the range of $0 \ldots 0.45$ with a step of 0.05 for a triangular cut, and Figure $5 \mathrm{~b}$ shows similar graphs of the change in curvature $f_{v} / l_{0}$ for a rectangular cutout. The permissible depth of the cut according to the graphs shown in Figure $5(a, b)$ is determined as follows. For example, you need to determine the permissible depth of a rectangular cutout with an initial curvature $f_{y}=5 \mathrm{~mm}$; $f_{x}=3 \mathrm{~mm}$ and the length of the bone $l_{0}=180 \mathrm{~mm}$ and allowable normal stresses $[\sigma]=12 \mathrm{MPa}(k=10)$ for a tensile load $P=15 \mathrm{~kg}$. Determine the relative initial curvature $f_{y} l_{0}=5 / 180=0.028 ; f_{x} l_{0}=3 / 180=0.017$. On the vertical axis, set the value of 0.028 and draw a horizontal line to the intersection with the graph (Fig. $4 \mathrm{~b})$ for $[\sigma]=2 \mathrm{MPa}(k=10)$. Draw a vertical line from the point of intersection down to the intersection with the horizontal axis. For a load of $15 \mathrm{~kg}$ and $[\sigma]=12 \mathrm{MPa}(k=10)$ for a given initial curvature of the bone, the relative depth of the rectangular cut should not exceed $h_{\delta} / H \leq 0.25$. Since the initial relative curvature $f_{x} / l_{0}=0.017$ falls into the range $0.01<f_{x} / l_{0}<0.02$, in which the two curves coincide, it is neglected.

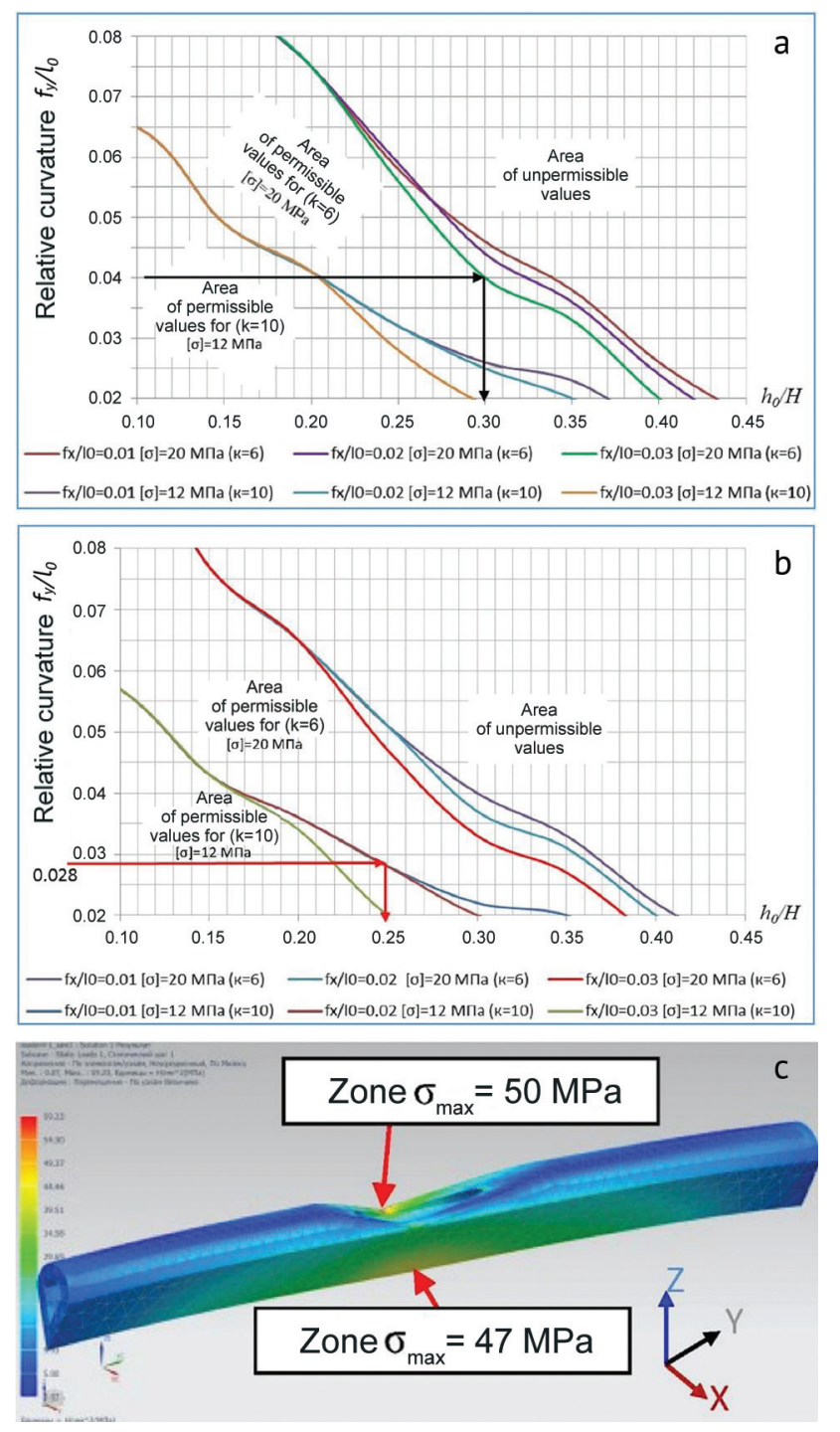

Fig. 5 Maximum permissible depth values: a triangular cut for the initial bone curvature $f / l$ from 0.02 to 0.08 ; $f / l$ from 0.01 to 0.03 at allowable stresses $[\sigma]=20 \mathrm{MPa}$ (with a safety factor $\mathrm{k}=6$ in relation to breaking stresses $\sigma_{v}=120 \mathrm{MPa}$ ) and $[\sigma]=12 \mathrm{MPa}$ (with a safety factor $k^{v}=10$ ) for tensile load $\mathrm{P}=15 \mathrm{~kg}$; b rectangular cut for the initial bone curvature $f_{y} / l_{0}$ from 0.02 to $0.08 ; f_{r} / l_{0}$ from 0.01 to 0.03 at permissible stresses $[\sigma]=20 \mathrm{MPa}$ (with a safety factor $k=6$ in relation to breaking stresses $\sigma_{v}=120 \mathrm{MPa}$ ) and $[\sigma]=12 \mathrm{MPa}$ (with $k=10$ ) for tensile load $\mathrm{P}=15 \mathrm{~kg}$; c stress distribution according to von Mises by loading the model with a triangular cut of depth $h_{0} / H=0.33$ and curvature $f_{x}$ and $f_{z}$ 
If it is necessary to determine the maximum permissible depth of the triangular cut of the bone with the parameters: initial curvature $f_{y}=7.2 \mathrm{~mm}$; $f_{x}=5.4 \mathrm{~mm}$ and the calculated bone length $l_{0}=180 \mathrm{~mm}$; section height $H=18 \mathrm{~mm}$ and permissible normal stresses $[\sigma]=20 \mathrm{MPa}(k=6)$ for a tensile load $P=15$ $\mathrm{kg}$, then we determine the relative initial curvature $f_{v}$ $l_{0}=7.2 / 180=0.04 ; f_{x} / l_{0}=5.4 / 180=0.03$. On the vertical axis, set the value of 0.04 and draw a horizontal line until it intersects with the graph $f_{x} / l_{0}=0.03$ (Fig. 5 a) for $[\sigma]$ $=20 \mathrm{MPa}(k=6)$. Draw a vertical line from the point of intersection down to the intersection with the horizontal axis. For a load of $15 \mathrm{~kg}$ and $[\sigma=0 \mathrm{MPa}(k=6)$ and a given initial curvature of the bone, the relative depth of the rectangular cut should not exceed $h_{\delta} / H \leq 0.3$.

As follows from the graph, with a load of $15 \mathrm{~kg}$ and $[\sigma]=12 \mathrm{MPa}(k=10)$, curvature in the $X O Z$ plane is 0.01 for a relative curvature in the $Y O Z$ plane of 0.02 , the relative permissible depth of a rectangular cut was 0.3 , for curvature 0.03 , respectively, 0.24 , in the conditions of curvature $0.04-0.16$, and for curvature $0.05-0.13$. Under similar conditions, for a triangular cut with curvature of 0.02 , the permissible depth of the cut was 0.37 , for curvature $0.03-0.26$, for $0.04-$ 0.21 , for $0.05-0.15$. Thus, the permissible depth of a rectangular cut, which is widely recommended in the literature, equal to $1 / 3$ of the bone diameter, can only be applied at a curvature of 0.02 . The permissible ratios of the value of the curvature and the depth of the cut during the formation of the graft should be in the permissible zone below the derived curves. When the depth of the cut exceeds the critical values, it becomes necessary to take measures to increase the strength of the radius.

The required dimensions of the section, initial curvature and calculated length are measured in X-ray images in two views and a CT image of the area of the graft collection and the entire forearm.

To evaluate and verify the results obtained by analytical methods, as well as to obtain new data, a calculation was performed using the numerical method on the developed geometric models (Fig. 5 c).

Resulting from the numerical analysis of stress fields, the following conclusions can be drawn:

- the curvature of the bone in two planes leads to its bending stresses in two planes;

- the formation of a cut always causes the formation of bending deformation, which depends on many factors;

- the magnitude of bending stresses significantly exceeds the stresses from central tension-compression;

- a triangular cut along the length has a significantly smaller zone of the highest stresses in comparison with a rectangular cut.

The values of the analytical data and the data of the numerical method turned out to be close to each other, what is a good result and indicates the adequacy of the models and the calculations performed.

Calculations on the computer models revealed that the structural shape of the cutout, which provides the greatest perception of the tensile load, is a triangular shape with a depth $\mathrm{h} 0 / \mathrm{H}=0.16$. The specimen with $\mathrm{fx}$ and $\mathrm{fz}$ curvature behaved the best way, which indicates the structural advantages of the bone with a natural curvature, rather than specimens without curvature or curvature in one of the axes (Table 1).

Numerical calculations have shown that the structural shape of the cutout, which provides the greatest perception of the torsional load, is a triangular shape for an artificial cutout with an immersion depth $h_{\delta} / H=0.16$ and a rectangular shape for an artificial cutout with an immersion depth $h_{\delta} / H=0.33$. The specimen without curvature behaved the best way, what indicates the structural defects of the bone with natural curvature by torsion. However, despite the greatest perception of the load of the bone model with a rectangular cut with a depth of immersion $h_{f} / H=0.33$, the model with a triangular cut has smaller displacements when a single moment is applied (Table 2).

Table 1

Stresses acting in specimens $\sigma \max \mathrm{MPa}$ in stretching the bone model with a cut depth of 0.16 and 0.33

\begin{tabular}{|l|c|c|c|c|c|}
\hline \multicolumn{1}{|c|}{ Type of curvature } & No cut & Rectangular, half & Rectangular full & Triangular, half & Triangular, full \\
\hline No curvature & 9.317 & 49.57 & 62.81 & 21.26 & 52.42 \\
\hline Curvature along $X f_{x}=3.73 \mathrm{~mm}$ & 24.44 & 58.91 & 98.4 & 34.6 & 95.27 \\
\hline Curvature along $Z f_{z}=6.38 \mathrm{~mm}$ & 31.59 & 37.26 & 47.4 & 35.88 & 45.35 \\
\hline Curvature $f_{x}=3.73 \mathrm{~mm}$ and $f_{z}=6.38 \mathrm{~mm}$ & 31.76 & 37.45 & 47.6 & 36.04 & 59.23 \\
\hline
\end{tabular}

Table 2

Stresses acting in specimens $\sigma \max \mathrm{MPa}$ by torsion of the bone model with a cut depth of 0.16 and 0.33

\begin{tabular}{|l|c|c|c|c|c|}
\hline \multicolumn{1}{|c|}{ Type of curvature } & No cut & Rectangular, half & Rectangular full & Triangular, half & Triangular,full \\
\hline No curvature & 0.77 & 6.091 & 6.081 & 2.83 & 16.25 \\
\hline Curvature along $X f_{x}=3.73 \mathrm{~mm}$ & 0.68 & 6.073 & 6.227 & 3.539 & 16.43 \\
\hline Curvature along $Z f_{z}=6.38 \mathrm{~mm}$ & 0.74 & 5.133 & 8.275 & 3.327 & 15.76 \\
\hline Curvature $f_{x}=3.73 \mathrm{~mm}$ and $f_{z}=6.38 \mathrm{~mm}$ & 0.757 & 5.136 & 7.759 & 2.825 & 14.13 \\
\hline
\end{tabular}


Analyzing the data obtained on computer models, we come to the conclusion that the model at a given load $\mathrm{P}=981 \mathrm{~N}$ cannot work without loss of stability in any type of artificial cut, since the critical stresses for the 1st type of stability loss are lower than the revealed maximum stresses at calculated load.

Calculations also showed that the structural shape of the plate enveloping the cylindrical surface of the bone has better load absorption both by torsion and tension than a narrow $10-\mathrm{mm}$ wide plate. As plate thickness increases up to $2 \mathrm{~mm}$, the load perception increases (Fig. 6). In tension, specimens with immersion of screws by $4 \mathrm{~mm}$ behaved best, while in torsion specimens with immersion of $4 \mathrm{~mm}$ and $13 \mathrm{~mm}$ behaved almost the same. The sample with throughout immersion of screws behaved the worst way and is not desirable for use, since in addition to poor load perception, this locking system is extremely traumatic for the patient. Analyzing the data, we come to the conclusion that the model with a metal compensating plate at a given load $\mathrm{P}$ can work without loss of stability in any type of artificial cut, since the critical stresses for the 1st type of stability loss are higher than the revealed maximum stresses at calculated load.

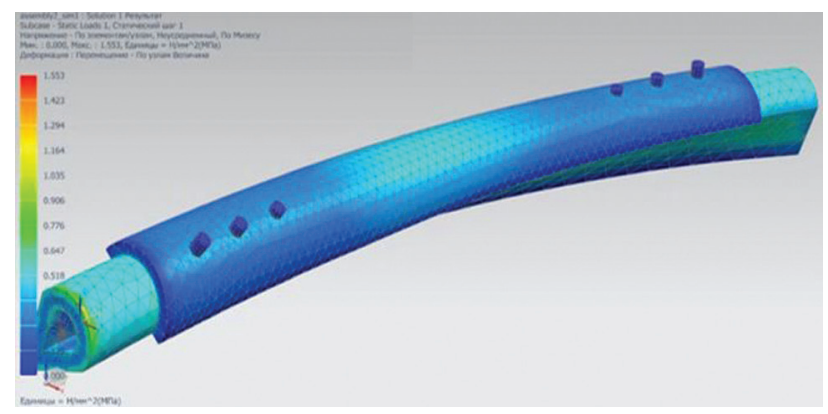

Fig. 6 Distribution of von Mises stresses in torsion of $\mathrm{M}=100 \mathrm{Nm}$ model with a triangular cut depth $h / H=0.33$, immersion of screws to $4 \mathrm{~mm}, 2-\mathrm{mm}$ plate thickness enveloping the cylindrical part of the bone

The modified plate proposed that envelops the cylindrical surface of the bone, reduces the level of stresses compared to a 10-mm wide flat narrow compensating plate by 1.2 times for an artificial rectangular cut, by 1.5 for an artificial triangular cut under tension, and by 3.9 times for a rectangular cutout, by 3.5 times for a triangular artificial cutout in torsion, and also increases the critical stresses by 1.5 times. By tension, the stress magnitudes correspond to the ones of an intact bone, while in torsion they are close and amount to $60-70 \%$ of the work of the intact bone. According to the stability criterion, the proposed plate has higher values than the intact bone.

\section{DISCUSSION}

The analysis of the literature shows that the problem of reducing the defect of the donor radius and preventing its fracture remains far from being resolved. Many issues of the problem remain unexplored. One of its main questions is to determine the strength properties of the donor radius after the collection (harvesting) of a marginal graft due to high incidence of its fractures. The dimensions of the marginal defect in the radius leading to a donorsite defect after graft collection depend on the graft size. We conducted thorough calculations of the strength of the intact and defective radius with marginal cutouts of various depths and shapes using mathematical and computer models obtained on the basis of experimental data on cadaveric bones under tensile, bending and torsion loads.

Mathematical calculation models of the bone were studied under a longitudinal force $\mathrm{N}=15 \mathrm{~kg} \approx 147$ $\mathrm{N}$, what corresponds to the average weight lifted by a person, as well as with known from the literature transverse physiological bending loads acting on the forearm. The permissible depth of the cut under the conditions of transverse physiological bending for a rectangular defect was 0.22 and 0.49 for a triangular defect. Thereby, the dangerous section of the rectangular cut is located in the action zone of a more significant bending moment and normal stresses. It indicates the effect of defect location on the strength of the bone. The maximum moment due to physiological efforts falls on the proximal third of the radius, and therefore the donor defect should not capture this area. Research has shown that a triangular cut is more beneficial than a rectangular cut in both tension-compression and torsion. The study of stress fields according to von Mises on computer volumetric models revealed that the magnitude of the stress concentration factor reaches a value of 1.2 in a rectangular cutout, and the maximum stress is $73 \mathrm{MPa}$. For a triangular cutout, the stress concentration decreases due to a smooth change in the cross-sectional areas and does not exceed $50 \mathrm{MPa}$.

Moreover, the zone of increased stresses is significantly reduced in comparison with the rectangular cutout. The calculated data on the permissible depth of a rectangular cut under physiological loads turned out to be noticeably lower than the experimental data known from the literature $[15,23,38]$. It is related to the fact that the authors of the works did not study the effect of defect location on bone strength. Only the calculated depth of a triangular cut exceeds the critical depth recommended in the literature, equal to $1 / 3$ of the bone diameter. Experimental and theoretical studies allowed us to identify previously unexplored factors affecting the strength of the radius with a marginal defect under these loads. It appeared that the strength of the bone depends not only on the depth of the cut, as stated in the literature and is currently accepted, but also on the nature of the load, the initial curvature of the bone, the shape of the cut and its location. We also investigated the combined effect of these factors on bone strength. Our study found that due to the initial curvature of the bone as well as as due to a marginal cutout, normal stresses always arise in the cross-sections of the bone (directed perpendicular 
to the cross-section and uniformly distributed over the section) under tensile load and bending stresses in two planes, which exceed tensile stresses in magnitude, as was shown in experimental studies [30], and can be decisive for breaking (fracture) of the bone due to tension or compression.

However, the grade of influence of these stresses on the strength of the bone has not been studied. There are no data on the permissible quantitative criteria for curvature and the depth of the cut. Only a few studies suggest the appearance of flexion force when the bone is stretched due to its curvature, but they could not quantitatively characterize these forces $[31,39]$. Using the developed mathematical and computational models, we studied the dependence of normal and bending stresses, as well as external breaking loads, and, consequently, the strength properties of the bone on the magnitude of its curvature, shape and depth of the defect. It was found that normal stresses increase not only with an increase in the depth of the cut, but also with the presence and increase in bone curvature. Moreover, the initial curvature of the bone has a more significant effect on the magnitude of the maximum normal stresses, external breaking loads than the presence of a cut notch. It was also revealed that the curvature of the bone in both of its planes affects the normal stresses. External breaking loads also depend on the curvature and, as it increases, decrease exponentially. It indicates a growing decrease in bone strength. In a combined effect of unfavorable factors on the radius, the probability of its fracture increases significantly.

We have also established for the first time that the magnitude of bone curvature increases dramatically in the presence of a marginal bone defect, since under tensile conditions it leads to an additional decrease in the strength of the bone already weakened due to the cutot. The effect of curvature on normal bone stresses is most pronounced with deep defects, since with the same curvature normal stresses greatly increase due to a reduction in the remaining part of the section. Moreover, not only the area decreases, but also the moments of inertia of this area. Therefore, bone curvature has a significant effect on the allowable cut depth. For this reason, if bone curvature is big, a fracture may occur at a cut depth of less than $1 / 3$ of the diameter, which is currently accepted in the literature as the critical one but bone curvature is not considered.

The simultaneous influence of bone curvature in two planes and a rectangular or triangular cutouts on normal stresses was also studied. It enables to determine the permissible cut depth. It has been established that in the case of a combined effect of unfavorable factors on the radius, the probability of its fracture increases significantly. On the basis of the identified dependencies, graphs were built that allow determining the critical depth of its cut considering the curvature in two planes. It was found that it is inappropriate to apply the recommended standard critical depth equal to $1 / 3$ of the bone diameter for all cases. The permissible depth of the cut should be determined individually for each patient, taking into account the bone curvature in two planes and the shape of the cut. It was found that, depending on the curvature of the bone, the permissible depth of the cut for graft collection will be different. At high magnitude of the curvature, the bone must be reinforced even at a cut depth of less than $1 / 3$ of the diameter. Low curvature allows a cut with a depth of more than $1 / 3$ of the diameter of the bone shaft to maintain its sufficient strength. Thus, depending on the curvature, the donor bone must be enhanced even if the defect depth is less than $1 / 3$ of the diameter, or it is allowed to exceed this magnitude without additional fixation of the bone.

In case of a forced or accidental increase in the critical depth of the cut or the defect located in the proximal half of the radius, it is necessary to perform (absolute indication) its preventive osteosynthesis. If the critical depth is not exceeded, then additional strengthening of the donor radius may be omitted. Currently, in order to prevent a fracture of the donor radius and by determining the indications for its preventive osteosynthesis, only the depth of the cut is considered. Preventive osteosynthesis and bone grafting of the defect is performed in cases when the depth of the cut exceeds $1 / 3$ of the diameter of the bone in its diaphyseal part. The study also enabled to substantiate rational options for marginal osteotomy and preventive osteosynthesis of the radius. It has been established by the method of computer modeling that the plate enveloping the cylindrical surface of the bone, fixed to it with six screws (three screws proximal and distal to the defect), has a better perception of the load both by torsion and by tension of the bone, if the immersion depth of the screws is $4 \mathrm{~mm}$.

Thus, the results obtained indicate that by planning the operations with the use of a osteocutaneous graft from the radius for various areas of the human skeleton, it is imperative to determine the curvature of the radius in two planes before the operation in order to establish the permissible cut depth. It is performed in plain radiographs and CT scans of the forearm bones in the frontal and lateral views. In the lateral radiograph of the entire forearm, it is possible to determine the bending of the radius in the YOZ plane, and in the direct view in the XOZ plane. The graphs of the dependence of destructive loads and normal stresses on the cut shape, depth, location and bone curvature are of practical importance, facilitate the surgical tactics for choosing additional methods to exclude the likelihood of a fracture or preventive strengthening of the donor bone in a particular patient and more clearly focus on the magnitude of the curvature as one of the main factors affecting the strength of the bone after the marginal cut.

Currently, there are several works in the available literature devoted to the study of the strength of long bones after resection due to a chronic inflammatory process [1]. The authors observed that defects in the diaphyseal part of the bone have a greater impact on the strength than defects in the metadiaphyseal part. It is also consistent with our results. The data obtained by us may be extrapolated to other long and short bones 
of the skeleton with a marginal defect after collecting a graft or marginal resection for any pathological process (inflammation, tumor, traumatic defect, systemic diseases, congenital malformations etc.), when the likelihood of a fracture in the area of intervention on the bone inevitably increases due to a decrease in its strength. By decision making on the need to strengthen the bone in such cases, it is also advisable to take into account all the identified factors affecting the strength of the radius with a marginal defect.

The results obtained also indicate that the programs of rehabilitation, kinesitherapy, household and professional loads after osteocutaneous grafting with a radial flap should be made taking into account the shape, location, depth of the cut, bone curvature in two planes and options for its strengthening, as well as stress-strain condition of the radius. The complex of rehabilitation measures should include active and passive movements in the joints of the fingers, wrist and elbow joints with a gradual increasing force immediately after the removal of sutures, relief of pain and reduction of edema, taking into account the arising maximum stresses in the bone until individual average loads are achieved similar to a healthy extremity. The average load on a healthy human bone depends on its anatomical, functional and anthropometric characteristics; and therefore should be determined individually before the start of rehabilitation measures as the average minimum and maximum loads. The average load on the operated forearm should be determined taking into account the ratio of the detected stresses in the intact and defective bones by specific movements.

This approach provides stabilization and reduction of normal stresses, deformation (sheer) of the bone in the defect area, which creates optimal conditions for reparative bone tissue regeneration, stability of the joint, restructuring of the bone graft, and prevention of fracture. Taking into account the acting stresses, regardless of the magnitude of the applied load, for the exercises that cause stretching or compression of the radius, in bone bending along the $\mathrm{X}$-axis for a rectangular cutout with a depth of 0.33 , the load should be reduced by 4 times in comparison with a healthy forearm, and for a rectangular cutout with a depth of 0.16 by 2.5 times. For a triangular cutout with a depth of 0.33 it should be also reduced by 4 times, and with a depth of 0.16 by 1.5 times. In the conditions of bone bending along the Z-axis, as well as along the $\mathrm{X}$ and $\mathrm{Z}$ axes, the load for both types of cuts with a depth of 0.33 should be reduced by 1.5 times, and at a depth of 0.16 it should be identical to that of a healthy forearm. Particular care should be taken by training forearm pronation-supination movements. Taking into account the normal stresses generated by application of single moment of force, for a rectangular cut of any depth under bending conditions along the $X$, $\mathrm{Z}$, as well as $\mathrm{Z}$ and $\mathrm{X}$ axes, the rotational loads should be reduced by 9.5 times, while for a triangular cut with a depth of 0.33 by 24 times, with the depth of 0.16 by 9 times compared to a healthy bone.

Similar ratios of loads should be for a triangular cut in bending along the $\mathrm{Z}$ axis, as well as the $\mathrm{Z}$ and $\mathrm{X}$ axes. Active and passive movements in the joints must be trained with increasing load, keeping the ratio of their magnitude for the operated and intact bone for each load value. The maximum loads should be allowed after the completion of reparative bone regeneration or consolidation and bone graft restructuring that takes about one year. In the case of preventive osteosynthesis, the load on the reinforced bone should be identical to that on the intact bone in extension and may be applied early after the operation. For rotational movements with the radius, the load should be $60-70 \%$ of the work of the intact bone.

The revealed patterns can also be used in practice in the development of various options for osteosynthesis of the radial bone, intact until the moment of injury, for fractures of all possible locations and types, after corrective osteotomies, wrist joint arthroplasty, as well as kinesitherapy programs individually for each patient.

\section{CONCLUSION}

The residual strength of the radius with a marginal defect depends on the cut depth, shape, location and the curvature of the bone in two planes. It should be considered by graft harvesting, as well as for its preventive and therapeutic osteosynthesis. A triangular cut is more preferred for load bearing perception. The critical depth of the cut should be determined taking into account its shape and the bone curvature in two planes, using the dependencies revealed above and individually for each patient. If the critical depth of the cut is exceeded, preventive osteosynthesis is indicated.

\section{Conflict of interest: not declared.}

\section{REFERENCES}

1. Alvi S.A., Hamill C.S., Lepse J.P., Ayala M., Girod D.A., Tsue T.T., Shnayder Y., Kakarala K. Outcomes after free tissue transfer for composite oral cavity resections involving skin. Head Neck, 2018, vol. 40, no. 5, pp. 973-984. DOI: 10.1002/hed.25062

2. Zenn M.R., Hidalgo D.A., Cordeiro P.G., Shah J.P., Strong E.W., Kraus D.H. Current role of the radial forearm free flap in mandibular reconstruction. Plast. Reconstr. Surg., 1997, vol. 99, no. 4, pp. 1012-1017. DOI: 10.1097/00006534-199704000-00014

3. Frederick J.W., Sweeny L., Carroll W.R., Peters G.E., Rosenthal E.L. Outcomes in head and neck reconstruction by surgical site and donor site. Laryngoscope, 2013, vol. 123, no. 7, pp. 1612-1617. DOI: 10.1002/lary.23775

4. Jones N.F., Jarrahy R., Kaufman M.R. Pedicled and free radial forearm flaps for reconstruction of the elbov, wrist, and hand. Plast. Reconstr. Surg., 2008, vol. 121, no. 3, pp. 887-898. DOI: 10.1097/01.prs.0000299924.69019.57

5. Kim S.K., Kim T.H., Yang J.I., Kim M.H., Kim M.S., Lee K.C. The etiology and treatment of the softened phallus after the radial forearm osteocutaneous free flap phalloplasty. Arch. Plast. Surg., 2012, vol. 39, no. 4, pp. 390-396. DOI: 10.5999/aps.2012.39.4.390

6. Shnayder Y., Tsue T.T., Toby E.B., Werle A.H., Girod D.A. Safe osteocutaneous radial forearm flap harvest with prophylactic internal fixation. Craniomaxillofac. Trauma Reconstr., 2011, vol. 4, no. 3, pp. 129-136. DOI: 10.1055/s-0031-1279675.

7. Sinclair C.F., Gleysteen J.P., Zimmermann T.M., Wax M.K., Givi B., Schneider D., Rosenthal E.L. Assessment of donor site morbidity for free radial forearm osteocutaneous flaps. Microsurgery, 2012, vol. 32, no. 4, pp. 255-260. DOI: 10.1002/micr.21950 
8. Satteson E.S., Satteson A.C., Waltonen J.D., Li Z., Wiesler E.R., Apel P.J., Graves B.R. Donor-Site Outcomes for the Osteocutaneous Radial Forearm Free Flap. J. Reconstr. Microsurg., 2017, vol. 33, no. 8, pp. 544-548. DOI: 10.1055/s-0037-1602740

9. Werle A.H., Tsue T.T., Toby E.B., Girod D.A. Osteocutaneous radial forearm free flap: its use without significant donor site morbidity. Otolaryngol. Head Neck Surg., 2000, vol. 123, no. 6, pp. 711-717. DOI: 10.1067/mhn.2000.110865

10. De Witt C.A., de Bree R., Verdonck-de Leeuw I.M., Quak J.J., Leemans C.R. Donor site morbidity of the fasciocutaneous radial forearm flap: what does the patient really bother? Eur. Arch. Otorhinolaryngol., 2007, vol. 264, no. 8, pp. 929-934. DOI: 10.1007/s00405-007-0277-1

11. Lipatov K.V., Gavriushenko N.S., Stan E.A. Obosnovanie vybora tipa khirurgicheskoi trepanatsii dlinnoi kosti pri lechenii bolnykh khronicheskim osteomielitom (eksperimentalnoe issledovanie) [Rationale for choosing the type of long bone surgical trepanation in the treatment of patients with chronic osteomyelitis]. Vestnik Travmatologii i Ortopedii im. N.N. Priorova, 2008, no. 3, pp. 62-66. (in Russian)

12. Connolly T.M., Sweeny L., Greene B., Morlandt A., Carroll W.R., Rosenthal E.L. Reconstruction of midface defects with the osteocutaneous radial forearm flap: Evaluation of long term outcomes including patient reported quality of life. Microsurgery, 2017, vol. 37, no. 7, pp. 752-762. DOI: 10.1002/micr.30201

13. Waits C.A., Toby E.B., Girod D.A., Tsue T.T. Osteocutaneous radial forearm free flap: long-term radiographic evaluation of donor site morbidity after prophylactic plating of radius. J. Reconsr. Microsurg., 2007, vol. 23, no. 7, pp. 367-372. DOI: 10.1055/s-2007-992342

14. Bardsley A.F., Soutar D.S., Elliot D., Batchelor A.G. Reducing morbidity in the radial forearm flap donor site. Plast. Reconstr. Surg., 1990, vol. 86, no. 2, pp. 287-294.

15. Thoma A., Khadaroo R., Grigenas O., Archibald S., Jackson S., Young J.E., Veltri K. Oromandibular reconstruction with the radial-forearm osteocutaneous flap: experience with 60 consecutive cases. Plast. Reconstr. Surg., 1999, vol. 104, no. 2, pp. 368-380. DOI: 10.1097/00006534-199908000-00007

16. Bowers K.W., Edmonds J.L., Girod D.A., Jayaraman G., Chua C.P., Toby E.B. Osteocutaneous radial forearm free flaps. The necessity of internal fixation of the donor-site defect to prevent pathological fracture. J. Bone Joint Surg. Am., 2000, vol. 82, no. 5, pp. 694-704.

17. Clark S., Greenwood M., Banks R.J., Parker R. Fracture of the radial donor site after composite free flap harvest: a ten-year review. Surgeon, 2004, vol. 2 , no. 5, pp. 281-286. DOI: 10.1016/s1479-666x(04)80098-2

18. Meland N.B., Maki S., Chao E.Y., Rademaker B. The radial forearm flap: a biomechanical study of donor- site morbidity utilizing sheep tibia Plast. Reconstr. Surg., 1992, vol. 90, no. 5, pp. 763-773.

19. Richardson D., Fisher S.E., Vaughan E.D., Brown J.S. Radial forearm flap donor-site complications and morbidity: a prospective study. Plast. Reconstr. Surg., 1997, vol. 99, no. 1, pp. 109-115. DOI: 10.1097/00006534-199701000-00017

20. Riecke B., Kohlmeier C., Assaf A.T., Wikner J., Drabik A., Catalá-Lehnen P., Heiland M., Rendenbach C. Prospective biomechanical evaluation of donor site morbidity after radial forearm free flap. Br. J. Oral Maxillofac. Surg., 2016, vol. 54, no. 2, pp. 181-186. DOI: 10.1016/j.bjoms.2015.11.021

21. Torina P.J., Matros E., Athanasian E.A., Cordeiro P.G. Immediate bone grafting and plating of the radial osteocutaneous free flap donor site. Ann. Plast. Surg., 2014, vol. 73, no. 3, pp. 315-320. DOI: 10.1097/SAP.0b013e31827a2fe4

22. Rebrov V.N., Gavriushenko N.S., Malygina M.A., Plotnikov S.Iu. Izuchenie prochnostnykh kharakteristik distalnogo metaepifiza luchevoi kosti i sistem «kost-fiksator» [Studying the strength characteristics of the distal radial meta-epiphysis and "bone-fixator" systems]. Vestnik Travmatologii $i$ Ortopedii im. N.N. Priorova, 2008, no. 2, pp. 57-60. (in Russian)

23. Swanson E., Boyd J.B., Manktelow R.T. The radial forearm flap: reconstructive applications and donor-site defects in 35 consecutive patients. Plast. Reconstr. Surg., 1990, vol. 85, no. 2, pp. 258-266.

24. Loeffelbein D.J., Al-Benna S., Steinsträßer L., Satanovskij R.M., Rohleder N.H., Mücke T., Wolff K.D., Kesting M.R. Reduction of donor site morbidity of free radial forearm flaps: what level of evidence is available? Eplasty, 2012, vol. 12, pp. e9.

25. Varghese B., Short D., Penmetsa R., Goswami T., Hangartner T. Computed- tomography-based finite-element models of long bones can accurately capture strain response to bending and torsion. J. Biomech., 2011, vol. 44, no. 7, pp. 1374-1379. DOI: 10.1016/j.jbiomech.2010.12.028

26. Ilichev N.A., Koliabin V.V., Kulepov V.F., Mikheev N.N., Naumov V.K. Opredelenie napriazhenii i raschety na prochnost sterzhnevykh sistem: ucheb. posobie [Determination of stresses and strength calculations of rod systems. Textbook]. Ed. by Iu.V. Gliavin. N. Novgorod, Nizhegorod. Gos. Tekhn. Un-t im. R.E. Alekseeva, 2009, 130 p. (in Russian)

27. Avery C.M., Bujtár P., Simonovics J., Dézsi T., Váradi K., Sándor G.K., Pan J. A finite element analysis of bone plates available for prophylactic internal fixation of the radial osteocutaneous donor site using the sheep tibia model. Med. Eng. Phys., 2013, vol. 35, no. 10, pp. 1421-1430. DOI: 10.1016/j.medengphy. 2013.03.014

28. Matsuura Y., Kuniyoshi K., Suzuki T., Ogawa Y., Sukegawa K., Rokkaku T., Thoreson A.R., An K.N., Takahashi K. Accuracy of specimen-specific nonlinear finite element analysis for evaluation of radial diaphysis strength in cadaver material. Comput. Methods Biomech. Biomed. Engin., 2015, vol. 18 , no. 16 , pp. 1811-1817. DOI: $10.1080 / 10255842.2014 .974579$

29. Karamanoukian R., Gupta R., Evans G.R. A novel technique for the prophilactic plating of the osteocutaneos radial forearm flap donor site. Ann. Plast. Surg., 2006, vol. 56, no. 2, pp. 200-204. DOI: 10.1097/01.sap.0000186859.91656.ca

30. Aleksandrov N.M., Veshutkin V.D., Zhukov A.E., Bashkalina E.V., Uglev O.I., Volkov G.A. Izuchenie prochnostnykh svoistv donorskoi luchevoi kosti raschetno-eksperimentalnym metodom [Studying the strength properties of the donor radius by the calculation-experimental method]. Rossiiskii Zhurnal Biomekhaniki, 2017, vol. 21, no. 2, pp. 147-165. (in Russian)

31. Shaaban H., Giakas G., Bolton M., Williams R., Wicks P., Scheker L.R., Lees V.C. The load-bearing characteristics of the forearm: pattern of axial and bending force transmitted through ulna and radius. J. Hand Surg. Br., 2006, vol. 31, no. 3, pp. 274-279. DOI: 10.1016/j.jhsb.2005.12.009

32. Zatsiorskii V.M., Aruin A.S., Seluianov V.N. Biomekhanika dvigatelnogo apparata cheloveka [Biomechanics of the human locomotor system]. M., Fizkultura i Sport, 1981, 143 p. (in Russian)

33. Korolev S.B., Nosov O.B., Klenin A.A., Veshutkin V.D. Sravnenie stabilnosti razlichnykh sposobov osteosinteza pri perelomakh golovchatogo vozvysheniia plechevoi kosti metodom matematicheskogo modelirovaniia [Comparison of the stability of various methods of osteosynthesis in fractures of the capitate eminence of the humerus by the method of mathematical modeling]. Fundamentalnye Issledovaniia, 2013, no. 9-3, pp. 375-379. (in Russian)

34. Harberkorn-Butendeich E. Die Kraft des Musculus triceps brachii. Diss. ... dr. med. [The power of the triceps brachii muscle. Dr. med. sci. diss.] Saarbrucken, 1973, 120 p. (in German)

35. Diakov I.F., Chernov S.A., Chernyi A.N. Metod konechnykh elementov v raschetakh sterzhnevykh sistem. Ucheb. posobie [The finite-element method in calculations of rod systems. Manual]. Ulianovsk, UlGTU, 2010, 133 p. (in Russian)

36. Zenkevich O. Metod konechnykh elementov v tekhnike [The finite-element method in engineering]. Ed. by Pobedria B.E. M., Mir, 1975, 542 p. (in Russian)

37. Bruiaka V.A., Fokin V.G., Kuraeva Ia.V. Inzhenernyi analiz v ANSYS Workbench. Ucheb. posobie. V 2 ch. [Engineering analysis in ANSYS Workbench. Manual. In 2 parts]. Samara, Samar. Gos. Tekhn. Un-t, 2010-2013. (in Russian)

38. Swanson E., Boyd J.B., Mulholland R.S. The radial forearm flap: a biomechanical study of the osteotomized radius. Plast. Reconstr. Surg., 1990, vol. 85 , no. 2 , pp. $267-272$.

39. Shaaban H., Giakas G., Bolton M., Williams R., Scheker L.R., Lees V.C. The distal radioulnar joint as s load-bearning mechanism - a biomechanical study. J. Hand Surg. Am., 2004, vol. 29, no. 1, pp. 85-95. DOI: 10.1016/j.jhsa.2003.10.020

\section{Received: 27.05 .2020}

\section{Information about authors:}

1. Nikolai M. Aleksandrov, M.D., Ph.D.,

Privolzhsky Research Medical University, Nizhny Novgorod, Russian Federation,

Email: aleksandrov-chetai@rambler.ru

2. Vladimir D. Veshutkin, Ph.D. of Engineering Sciences, Nizhny Novgorod State Technical University named after R.E. Alekseev, Nizhny Novgorod, Russian Federation, Email: vveshutkin@mail.ru

3. Aleksandr E. Zhukov, Ph.D. of Engineering Sciences, Nizhny Novgorod State Technical University named after R.E. Alekseev, Nizhny Novgorod, Russian Federation
4. Ivan D. Veshaev, M.D.,

Privolzhsky Research Medical University, Nizhny Novgorod, Russian Federation

5. Dmitriy A. Kuptsov, M.D.,

Privolzhsky Research Medical University, Nizhny Novgorod, Russian Federation

6. Oleg I. Uglev, M.D., Privolzhsky Research Medical University, Nizhny Novgorod, Russian Federation 